\title{
The Relevance of the Teaching of Astronomy in the Developing Countries; a Case: Paraguay
}

Alexis E. Troche-Boggino

Instituto de Ciencias Basicas, Universidad Nacional de Asuncion, Asuncion, Paraguay

Last year, I enjoyed having dinner with an American astronomer who was visiting my country. He mentioned that astronomy is an active science that is an expensive and luxury enterprise, and one for which it is difficult to receive government support because other needs have higher priority. He works in a state like my country, where cattle and agriculture are the most important products. I answered him as follows: Scientific research - particularly astronomy, of course - requires much effort, skill, and a great deal of economic support, but there are many young, bright students in my country, as everywhere, who have the potential to develop fully, provided that challenges are offered to them.

I would like to remind you about some famous astronomers who became IAU Presidents: the late Dr. Bappu from India and the current President, Dr. Sahade from Argentina; also, one of the present Vice-Presidents, Dr. Peimbert, is from México. These astronomers have come from developing countries. Other first-rate astronomers from these countries have helped educate young astronomers and build astronomical institutions of research. It is worthwhile to mention here Dr. Guillermo Haro - a Mexican astronomer who passed away just a few months ago - who made several contributions to astronomical research, especially the discovery of HerbigHaro objects. Also, astronomers from countries where astronomy is fully developed have done much for astronomy education, observatories and research institutes in third world countries.

Further, I would like to mention two IAU programs that are helping a lot in some countries. These are: a) The International Schools for Young Astronomers (ISYA), under the secretariat of Dr. Kleczek. I am particularly grateful to this program, being myself twice a student of it and having been helped to become an IAU member, initially as a Commission 46 consulting member. b) The Visiting Lecturers Program (IAU Commissions 38 \& 46). Dr. Donat Wentzel, then president of IAU Commission 46, proposed this program, which is doing a lot for astronomy in Peru, Nigeria, and since last April in Paraguay. In this program 20 students from the two Paraguayan universities, the Universidad Nacional de Asunción and the Universidad Católica N.S. de la Asunción became associated in the VLP. I hope that this program will enable our young scientists to work professionally in the field of astronomy with opportunities of collaboration with colleagues from other countries in the future. There are plans to cooperate with the Argentinians in verylong-baseline radio interferometric observations. The economic support to continue with the VLP program for the next term is being sought and your advice about the sources for building a radiotelescope are welcome.

I would like to quote here the words of Dr. Richard West, past IAU General Secretary, during the last General Assembly at Delhi in 1985 about future directions for the IAU: "There are two kinds of activities, perhaps in every scientific union, 
which are important. The IAU has put much emphasis on one of these all the time... The first is the question of top science, frontline science, having scientific meetings, symposia, colloquia, regional meetings, and by having General Assemblies you promote top science. But on the question of science in the developing countries, if I may put it that way, or science for the young people, perhaps there has been a little less emphasis in the IAU in the early days. We now see that this comes more into the picture."

I am now thinking of another answer to the question of the relevance of teaching astronomy in developing countries. This question is about the need for astronomy for the sake of the people; or, in different words, the human side of astronomy. Most of us agree about the contributions provided through astronomy education besides answering people's curiosity about space and the universe. These contributions are: a) learning about the scientific method, in which astronomy serves so well; $b$ ) displaying the interdisciplinary relationships in which astronomy is doing a beautiful job, combining the efforts of physicists, chemists, biologists, historians, and others. It is possible to provide teachers from these different fields with good examples in which astronomy is involved. This possibility has been pointed out several times by a leader in the teaching of astronomy in Commission 46, Dr. Josip Kleczek. c) Another important point that IAU President Dr. Sahade has pointed out and which should be kept in mind is the importance of applied science and the transfer of technology, two major topics of great interest to developing countries. Dr. Sahade has said, "It is a mistake to think that the application of science and the transfer of technology alone can solve the problems of underdevelopment. The transfer of technology, to be efficient, needs a medium which provides scientific and technological potential, which should be able to take advantage of and use as a starting point to develop its own resources, for the future. Otherwise underdevelopment and dependence will remain and worsen." The progress of astronomy is an indicator of improvement.

I would like to stress other subtle points concerning the interaction between the decision-making countries and countries that follow the decision makers. I shall point out only a few aspects, the most relevant ones:

a) National security (meaning defense of democracy, territorial defense, and maintaining cultural and social values).

b) Human rights (as declared by the U.N.).

c) Environmental problems and the saving of natural resources and energy.

These policies being applied equally, by both types of countries should help them grow harmoniously both internally and externally, and thereby to end wars, poverty, and other human misery. But unfortunately these aims are seldom achieved. The enemies are ignorance, vanity, and selfishness. It is, of course, utopian to hope to cure these illnesses rapidly. Now astronomy might do a lot for our young people, to help them to have decent ideals, and to build up more sensibility towards human beings and nature. There is a great need for sensitive people from the two types of countries to cooperate in looking for solutions to the problems, and building up a 
more humane society. Remember that nations' attitudes are just the same as the attitudes of its individual members. I am sure that most of us present here have a positive attitude. So, let us make known our efforts and our science, to improve humanity. I feel strongly impelled to do so.

\section{Educational Material in Developing Countries}

The panel discussion on textbooks stimulated a great deal of interest and discussion, especially about the problem of educational material in and for the developing countries. About 50 participants in the Colloquium therefore reconvened one afternoon for an impromptu open discussion of this problem. the following notes were compiled by John R. Percy; they are based on the comments and suggestions of many participants.

\section{Acquiring Current Material from Abroad}

Current monographs and journals are essential for graduate teaching and research, and for the "professional development" of instructors, but there are major problems resulting from currency shortages or restrictions. Institutions must therefore be particularly selective in choosing material. IAU Commission 46 produces a triennial listing of astronomy education material in all major languages to help to deal with this problem. Institutions in developing countries can obtain some periodicals through exchange which institutions elsewhere, but problems of language, content, and shipping costs make this difficult. Another approach might be to "twin" institutions in the developing and the developed countries.

\section{Acquiring Less-Current Material from Abroad}

Several organizations are now engaged in collecting unused books and journals, and sending them to developing countries ${ }^{3}$. Publishers might be also induced to donate material, such as slightly-outdated editions of textbooks. In North America, new editions of introductory astronomy textbooks are introduced approximately every three years!

\section{Producing Translations of Important Material}

Translations of important and popular material could be made in the language of the developing country (and then shared by other countries with the same language). This makes good use of good material, but requires the cooperation of the author and publisher of the original edition. It also requires a good translator, who is willing to do it as a "labor of love": there is not much profit to be made in translating the average astronomy book! In the case of elementary and secondary school

\footnotetext{
${ }^{3}$ See the article by Batten and Brückner elsewhere in these proceedings.
} 